\title{
Análisis cuantitativo y cualitativo de la semipresencialidad del sistema universitario de Cataluña
}

\section{Quantitative and qualitative analysis of Blended Learning in the university system of Catalonia}

\author{
Joan Simon Pallisé \\ Carles Benedí González \\ Cèsar Blanché Vergés \\ Maria Bosch Daniel \\ Mercedes Torrado Fonseca \\ Universitat de Barcelona, UB (España)
}

\section{Resumen}

En los últimos años se ha producido un rápido crecimiento en la implementación del Blended Learning o aprendizaje combinado como modalidad docente, así como en la investigación de las metodologías relacionadas con las mejoras en los resultados de aprendizaje. Sin embargo, en universidades no anglosajonas son escasas las investigaciones sobre la política institucional vinculada a este tipo de docencia. En dos trabajos anteriores, los autores han publicado resultados previos sobre esta modalidad, a nivel general, referidos a 20 universidades europeas; así como un estudio en profundidad a nivel de la Universidad de Barcelona. En esta investigación se analiza la implementación actual de la semipresencialidad en las doce universidades integradas en el sistema universitario de Cataluña (NE de España) con el objetivo de identificar los elementos clave que determinan la adopción estratégica de esta modalidad. Para tal fin, se realizaron encuestas semiestructuradas a los doce vicerrectores/ as responsables de política docente en cada universidad. En el presente artículo se describe el estado actual del aprendizaje combinado en el sistema universitario catalán y se analiza en comparación a los datos de hace seis años. Se evidencia, por un lado, como en el nivel de grado, el aumento no ha sido el esperado con respecto a las expectativas previas. Por otro lado, se hace patente el cambio conceptual de la docencia semipresencial reglada, con acreditación de las autoridades académicas, hacia nuevos enfoques más flexibles de aprendizajes híbridos o combinados.

Palabras clave: semipresencialidad; metodologías híbridas; adopción institucional; política de educación superior. 


\begin{abstract}
In recent years, there has been a rapid growth in the implementation of Blended Learning as a teaching modality, as well as in the research of methodologies related to improvement in learning outcomes. However, in non-Anglo-Saxon universities, research on the institutional policy related to this type of teaching is scarce. In two earlier studies, the authors have published previous outcomes on this modality, at a general level, referring to 20 European universities, as well as an in-depth study at the University of Barcelona. This research analyzes the current implementation of Blended Learning in the twelve universities integrated in the university system of Catalonia (NE Spain) in order to identify the key elements that determine the strategic adoption of this modality. With this aim, thus, semi-structured surveys were carried out to the twelve vice-rectors responsible for educational policy in each university. This article describes the current state of the Blended Learning in the Catalan university system and it is analyzed in comparison to the data of six years ago. It is evident, on the one hand, as at the grade level, the increase has not been expected in relation to previous expectations. On the other hand, it becomes clear the conceptual change of the formal blended teaching, with the accreditation of academic authorities, towards new, more flexible approaches to hybrid or combined learning.
\end{abstract}

Keywords: blended learning; hybrid methodologies; institutional adoption; higher education policy.

Durante la mayor parte del siglo XX, se ha asociado el proceso de enseñanzaaprendizaje en la universidad con la presencia física en las aulas, libros de texto y exámenes finales. Sin embargo, las TIC han transformado irremisiblemente el aprendizaje tradicional presencial hacia el concepto de e-learning. El e-learning "puro" no parece ofrecer suficiente compromiso, opciones, relevancia, contacto social y contexto para facilitar el aprendizaje eficaz, efectivo y exitoso (Bartolomé, 2004). Una de las soluciones para este hándicap es la introducción de la modalidad del Blended Learning o aprendizaje combinado (AC) en el que se mezclan lo mejor de los dos enfoques de aprendizaje para cumplir objetivos educativos específicos (Graham, 2006; Porter, Graham, Spring y Welch, 2014).

El AC se está implantando en las universidades desde la última década del siglo XX (Garrison y Kanuka, 2004; Osguthorpe y Graham, 2003) especialmente en el ámbito anglosajón (Halverson, et al., 2014). El último informe de NMC-Horizon Report (Adams Becker et al., 2017) identifica el AC como el único ítem que se ha mantenido consecutivamente como tendencia en los últimos seis años. Según Norberg, Dzuban y Moskal (2011), el AC se intuye como la "nueva normalidad" o el "nuevo modelo tradicional" en educación universitaria (Ross y Gage, 2006).

El enfoque tradicional del aprendizaje ya no es ideal para la mayoría de estudiantes (Entwistle y Ramsden, 2015). El AC se considera un enfoque mejor, ya que conceptualiza el aprendizaje como un proceso continuo en lugar de un evento de tiempo único, e induce a los estudiantes a ser proactivos fuera del aula (Eddy, 
Nor-Aziah y Jasmine, 2014). En general, se admite que el AC no solo mejora el acceso, la flexibilidad y la metodología docente, sino también el compromiso, la interacción y la participación de los estudiantes (Alebaikan y Troudi, 2010; Garrison y Vaughan, 2007; Gómez e Igado, 2008; Graham, Allen y Ure, 2005; Korr, et al., 2012). Sin embargo, diversos estudios siguen mostrando que los académicos en general, y los profesores seniors en particular, se muestran aprensivos sobre el AC (Brooks, 2008; Moskal, Dziuban y Hartman, 2013; Porter y Graham, 2015). Entre los desafíos a superar para la introducción de soluciones combinadas en la educación superior, destaca la falta de recursos en un contexto de crisis, su relativa complejidad metodológica y las limitaciones institucionales (The Oxford Group, 2013).

A pesar del papel fundamental de los responsables académicos institucionales en el éxito de los esfuerzos de implementación del AC en la universidad, se ha publicado poco sobre las limitaciones y los obstáculos en su adopción por parte de los gestores de política universitaria (Abrahams, 2010). Sólo algunas investigaciones en Francia (Humbert, 2007), Corea (Oh y Park, 2009) y EUA (Graham, Woodfield y Harrison, 2012; Porter, Graham, Bodily y Sandberg, 2016) acometen esta tarea de identificar y explorar dichos factores.

En este contexto, los objetivos de la presente investigación han sido:

- Identificar y caracterizar la docencia semipresencial actual en las 12 universidades del sistema universitario de Cataluña y valorar su evolución en los últimos años.

- Determinar y analizar los factores fundamentales en su nivel de implantación y la valoración que hacen los responsables académicos.

- Esbozar hacia donde evoluciona la docencia semipresencial universitaria en el marco de las nuevas tendencias educativas en este ámbito.

\section{Antecedentes en nuestro entorno}

En el marco del arco mediterráneo europeo, cabe destacar los trabajos de Benítez y Aguilar (2010) para las universidades públicas de Andalucía; de Simón, Benedí y Blanché (2013) para 20 universidades del SO de Europa, y los de Expósito y Turrull (2014) y Simón, Benedí, Blanché y Bosch (2016) para la Universidad de Barcelona, todos ellos relacionados con la modalidad semipresencial universitaria. De los trabajos mencionados, se deduce que las principales preocupaciones y desafíos sobre esta modalidad están en: 1) la disminución de la calidad en la interacción con el estudiante, 2) la falta de tiempo del profesorado para preparar contenidos y actividades en línea, 3) la dificultad de interacciones en línea y sobrecarga de trabajo, 4) la falta de habilidades pedagógicas y técnicas para implementar esta modalidad y 5) la falta de financiación para formar equipos docentes potentes. En cuanto a los facilitadores, destacan: 1) la mejora en el proceso de aprendizaje de los estudiantes, 2) las ventajas metodológicas sobre la mejora de la enseñanza tradicional y 3) la mayor flexibilidad para los estudiantes. 


\section{Evolución y conceptualización de la semipresencialidad}

El concepto Blended Learning se ha traducido en un sinfín de términos: "semipresencialidad", "aprendizaje combinado", "aprendizaje híbrido", "aprendizaje mixto" o "instrucción tecnológica", entre otros. Igualmente, las dificultades de su conceptualización se han ido evidenciando (Simón, Benedí y Blanché, 2013, pp. 34-35). Actualmente, expertos como Graham (2013) sugieren que el aprendizaje combinado designa un amplio espectro de posibilidades, que combina Internet y medios digitales, con metodologías de aula más tradicionales que requieren la presencia física del profesor y los estudiantes.

Las posibilidades que ofrece el AC en cuanto a su flexibilidad, facilidad de acceso, integración de elementos multimedia y tecnologías sofisticadas, están profundamente estudiadas (Garrison y Vaughan, 2007; Kim, Bonk y Oh, 2008). El enfoque actual obvia cada vez más la reducción de las clases presenciales y se dirige más hacia la adaptación de las experiencias de aprendizaje para satisfacer las necesidades individuales de los estudiantes (Owston, York y Murtha, 2013) o, incluso, hacia "el aprendizaje personalizado" (Waha y Davis, 2014).

Las últimas tendencias sobre esta modalidad apuntan hacia enfoques cada vez más amplios dentro del concepto híbrido del Blended (Spring, Graham y Hadlock, 2016). Los avances en el Learning Analytics (análisis del aprendizaje), el Adaptative Learning (aprendizaje adaptativo) y una combinación de herramientas síncronas y asíncronas de vanguardia están empezando a desplegar y mejorar el aprendizaje combinado (Dziuban, 2015; Yamagata-Lynch, 2014).

Consideramos aquí el concepto de AC como la mezcla explícita de metodologías de aprendizaje, tanto presenciales como mediadas por la tecnología, que conlleva integrar la enseñanza basada en Internet con la interacción presencial del profesorado con su alumnado. Nos alejamos, pues, del concepto de semipresencialidad con carácter normativo (que abreviamos como SP) de la Agencia de Calidad del sistema universitario catalán (AQU) ligado a una presencia mucho menor del alumno en clase (entorno al 30\%) y que se relacionaba más estrechamente con el e-learning.

\section{METODOLOGÍA}

\section{Contexto de la Investigación}

Para poder identificar los elementos clave que determinan la adopción estratégica del AC en la educación superior, se plantea una investigación de corte descriptivo. El ámbito del estudio han sido las doce universidades que integran el sistema universitario de Cataluña (tabla 1 y figura 1), con una oferta de unos 1.300 títulos entre grados, másteres y doctorados, 26.300 docentes y 237.000 estudiantes. 
Figura 1. Ubicación de las 12 universidades objeto del estudio

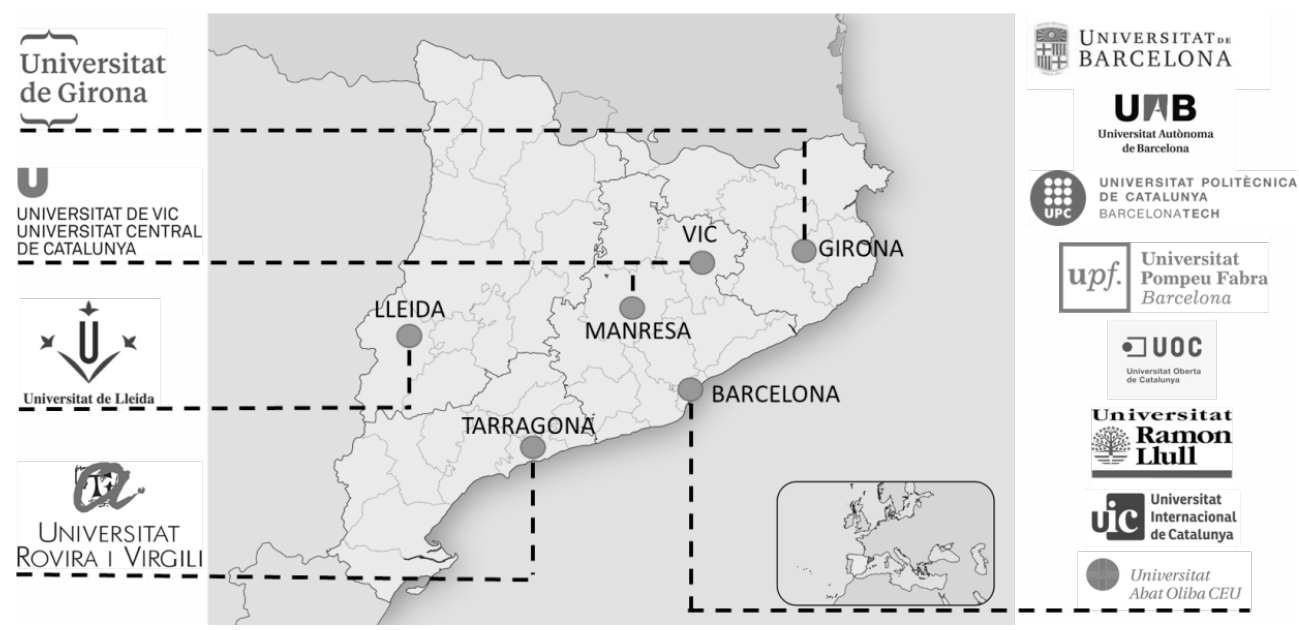

Tabla 1. Universidades estudiadas con sus siglas, el carácter público o privado y presencial o en línea

\begin{tabular}{|l|c|c|c|}
\hline \multicolumn{1}{|c|}{ NOMBRE } & SIGLAS & $\begin{array}{c}\text { PÚBLICA/ } \\
\text { PRIVADA }\end{array}$ & $\begin{array}{c}\text { PRESENCIAL/ } \\
\text { EN LINEA }\end{array}$ \\
\hline Universitat Abat Oliba & UAO & Privada & Presencial \\
\hline Universitat Autònoma de Barcelona & UAB & Pública & Presencial \\
\hline Universitat de Barcelona & UB & Pública & Presencial \\
\hline Universitat de Girona & UdG & Pública & Presencial \\
\hline Universitat Internacional de Catalunya & UIC & Privada & Presencial \\
\hline Universitat de Lleida & UdL & Pública & Presencial \\
\hline Universitat Oberta de Catalunya & UOC & Pública* & En línea \\
\hline Universitat Politècnica de Catalunya & UPC & Pública & Presencial \\
\hline Universitat Pompeu Fabra & UPF & Pública & Presencial \\
\hline Universitat Ramon Llull & URL & Privada & Presencial \\
\hline Universitat Rovira i Virgili & URV & Pública & Presencial \\
\hline $\begin{array}{l}\text { Universitat de Vic-Universitat Central } \\
\text { de Catalunya }\end{array}$ & UVic-UCC & Privada & Presencial \\
\hline
\end{tabular}

* Universidad con financiación pública, pero con estructura de gestión privada. 


\section{Recogida de información}

En primer lugarypara poder hacer una descripción contextual delas universidades y titulaciones implicadas, se consultó la información pública y disponible de la universidad a partir del Registre Oficial de Centres $i$ Estudis a Catalunya (ROC, 2016). En segundo lugar, se realizaron doce entrevistas semiestructuradas presenciales en el mes de febrero del 2017 a los doce vicerrectores ${ }^{1}$ con competencias en política docente.

Siguiendo estudios previos (Simón, Benedí y Blanché, 2013; Simón, Benedí, Blanché y Bosch, 2016) la entrevista ${ }^{2}$ se diseñó con asesoramiento del ICE-UB, con un total de 43 preguntas cerradas y 7 abiertas, permitiendo en su conjunto conocer la realidad de cada universidad participante en cuanto a:

La situación de la implementación de la semipresencialidad en los grados universitarios actuales, así como su evolución en los cinco últimos años. En esta primera parte se utilizaron 28 ítems de respuesta cerrada de elección única (afirmativa o negativa) para identificar la situación en relación a los tres aspectos: estrategia, estructura y soporte definidos por Graham, Woodfield y Harrison (2012) y posteriormente desarrollados por Porter et al. (2016).

Los factores clave que inciden o pueden explicar, solos o de forma combinada, el escaso avance de la semipresencialidad en los grados universitarios. En la segunda parte, se incluyeron 15 ítems de respuesta cerrada de escala numérica (del $1 \mathrm{al} 7$, de desacuerdo a muy de acuerdo). También se incluyó una pregunta abierta para poder explicar otros aspectos que dificultan o facilitan la promoción de la docencia semipresencial.

Aspectos determinantes que puedan establecer a corto plazo la introducción o implementación del AC, entendidas como evolución del concepto tradicional de docencia semipresencial. Se plantearon 7 preguntas abiertas.

Las entrevistas fueron presenciales, tuvieron una duración de 45-6o minutos y fueron grabadas previo consentimiento; al final de la conversación, los entrevistados proporcionaron su información demográfica. Las preguntas cerradas se analizaron con el SPSS-Win y en el caso de las preguntas abiertas se procedió a un análisis de contenido.

\section{RESULTADOS}

\section{Implementación de la semipresencialidad reglada}

Según los datos del ROC, en el cuso 2016-17 se han programado en el territorio estudiado un total de 19 grados $^{3}$ en modalidad semipresencial reglada, en los términos definidos por la $\mathrm{AQU}$, sobre un total de 484 grados con matrícula activa, lo que representa un $3,73 \%$ de la oferta de grado.

En la tabla 2 se ofrecen sintéticamente los datos comparativos de la oferta de grado actual con la vigente de hace cinco años, que nos permiten describir con 
bastante precisión la evolución de la semipresencialidad en el sistema universitario catalán. Así, la oferta de grados semipresenciales del curso 2016-17 proviene mayoritariamente de las universidades privadas (78\% de la oferta SP, mientras que su contribución conjunta a la oferta total de grados es inferior al 19\%), para las cuales significa una parte sustancial de sus grados (UAO: $27 \%$; UVic-UCC: $30 \%$; UIC: $8 \%$; URL: $3 \%$ ), frente a las universidades públicas (más del 80\% de las titulaciones de grado ofrecidas y el grueso del total de alumnos del sistema) para las que representa porcentajes mucho más bajos de su oferta (o-4\%) y, en varios casos, nunca se ha llegado a programar ningún grado SP (UAB, UdL, UdG). Además, hay que considerar que, en las públicas, de los cuatro únicos grados SP ofrecidos, tres de ellos lo son en centros adscritos (que pertenecen al sistema público por motivos administrativos, pero con una gestión muy próxima a la de entidades privadas), por lo que tampoco UPF ni UPC han programado grados públicos SP, que se reducen, en realidad, a uno solo en la UB.

La evolución en los cinco últimos años (tabla 2) nos confirma que las proporciones público/privado de oferta de grados SP se mantienen inalterables por lo que puede considerarse como una característica ya consolidada del sistema. La UVic-UCC es la que realiza una apuesta más decidida por la SP (el peso en su oferta de grado ha pasado del $14 \%$ al $30 \%$, aunque en las otras universidades privadas también se produce incremento, especialmente en la UAO) y representa el 50\% de la SP en grado en Cataluña.

Tabla 2. Grados en modalidad semipresencial en Cataluña en los cursos 2012-13 y 2016-2017

\begin{tabular}{|c|c|c|c|c|c|}
\hline \multirow{2}{*}{ Universidad $^{*}$} & \multirow{2}{*}{ Titular $^{1}$} & \multicolumn{2}{|c|}{ 2012-2013 } & \multicolumn{2}{c|}{ 2016-2017 } \\
\cline { 3 - 6 } & & N $^{0}$ grados SP & $\%$ total grados & $N^{0}$ grados SP & $\%$ total grados \\
\hline UAO & PRI & 2 & 15 & 3 & 27 \\
\hline UVic-UCC & PRI & 4 & 14 & 9 & 30 \\
\hline UIC & PRI & 1 & 7 & 1 & 8 \\
\hline URL & PRI & 0 & 0 & 1 & 3 \\
\hline UAB & PUB & 0 & 0 & 0 & 0 \\
\hline UB & PUB & 1 & 0,6 & 1 & 1,4 \\
\hline UdG & PUB & 0 & 0 & 0 & 0 \\
\hline UdL & PUB & 0 & 0 & 0 & 0 \\
\hline URV & PUB & 0 & 0 & 0 & 3 \\
\hline UPC & PUB & 1 & 1,6 & $2^{*}$ & 3 \\
\hline UPF & PUB & 0 & 0 & $1^{*}$ & 4 \\
\hline UOC & PUB & 0 & 0 & $1^{* *}$ & 0 \\
\hline
\end{tabular}

${ }^{1}$ Titularidad: PUB = Pública; PRI = Privada. ${ }^{*}$ Impartido en centros adscritos. ${ }^{*}$ Compartido con UVic-UCC.

Fuente: Elaboración a partir de Simon et al. (2013) y ROC (2016). 
En relación a la distribución de la oferta semipresencial en grado respecto a las ramas de conocimiento (Tabla 3), en la oferta para el curso 2016-17 se observa un claro predominio de la rama de Ciencias sociales y jurídicas en la oferta tanto de grados (73\%) como de másteres $(60,9 \%)$, seguida de Ciencias de la salud (20\% y $15,9 \%)$. En las tres ramas restantes, la oferta es nula o casi en grados, y en másteres comparativamente mucho menor.

Tabla 3. Comparación en 2016-17 de la oferta de grados y másteres semipresenciales agrupados según la rama de conocimiento

\begin{tabular}{|l|c|c|}
\hline \multicolumn{1}{|c|}{ Rama de conocimiento } & Grados 2016-2017 & Másteres 2016-2017 \\
\hline Ciencias sociales y jurídicas & $11(73,3 \%$ & $42(60,9 \%)$ \\
\hline Ciencias de la salud & $3(20 \%)$ & $11(15,9 \%)$ \\
\hline Ciencias & o (0\%) & $3(4,3 \%)$ \\
\hline Ingeniería y Arquitectura & o (o\%) & $9(13 \%)$ \\
\hline Artes y Humanidades & $1(6,7 \%)$ & $4(5,8 \%)$ \\
\hline
\end{tabular}

Fuente: ROC (2016).

Si se analiza la oferta de másteres ${ }^{4}$ en modalidad SP (Tabla 4), destaca la UVicUCC con más de la mitad de la oferta (57\%) en formato semipresencial, lo que se correlaciona con su también abundante oferta de grados en esta modalidad. La siguen con cerca de un 30\% la UAO y la UdL, siendo la oferta en el resto de universidades muy baja $(1,6-7,7 \%)$ o nula en UOC y UdG.

Tabla 4. Másteres activados el curso 2016-17, con la oferta total, semipresencial y porcentaje

\begin{tabular}{|c|c|c|c|}
\hline Universidad & $\begin{array}{c}\text { Másteres } \\
\text { Oferta total }\end{array}$ & $\begin{array}{c}\text { Másteres } \\
\text { Oferta modalidad } \\
\text { semipresencial }\end{array}$ & Másteres (\%) \\
\hline UAO & 11 & 3 & $27,3 \%$ \\
\hline UAB & 100 & 5 & $5,0 \%$ \\
\hline UB & 132 & 9 & $6,8 \%$ \\
\hline UdG & 30 & 0 & $0 \%$ \\
\hline UdL & 36 & 12 & $33,3 \%$ \\
\hline UVic-UCC & 14 & 8 & $7,1 \%$ \\
\hline UIC & 13 & 1 & $0 \%$ \\
\hline UOC & 27 & 10 & $1,6 \%$ \\
\hline UPC & 62 & 2 & $3,3 \%$ \\
\hline UPF & 60 & 4 & $7,4 \%$ \\
\hline URL & 54 & 10 & $2,5 \%$ \\
\hline URV & 40 & 0 & \\
\hline
\end{tabular}

Fuente: ROC (2016). 


\section{Estado de implantación y desarrollo institucional}

En general, no se dispone de documentos específicos sobre organización y estrategia relativa a la SP y, si los hay, forman parte de directrices o textos de foco más amplio y que abarcan otras modalidades docentes; en algún caso se han creado comisiones o grupos de trabajo específicos a escala de universidad, en general de carácter exploratorio. Raramente se dispone de personal técnico de referencia en SP dedicado en exclusiva, y se recurre al apoyo técnico genérico, que se considera suficiente. Tan solo 3 universidades tienen previsto instaurar nuevos grados semipresenciales en el próximo curso: UVic-UCC (uno, ya catalogado en el registro ROC), UdL y UPF (sin precisar).

Las respuestas abreviadas y cuantitativas de la entrevista sobre los ítems del avance de la implantación de la SP en las universidades encuestadas se muestran en la Tabla 5, aunque su interpretación completa requiere ser complementada con los comentarios disponibles en las grabaciones.

Tabla 5. Resumen de la opinión de los vicerrectores entrevistados al cuestionario sobre características de la implantación de grados SP en las universidades analizadas

\begin{tabular}{|l|l|l|l|l|l|l|}
\hline \multicolumn{5}{|c|}{ Características de la implantación de SP en los grados } \\
\hline \multicolumn{1}{|c|}{$\begin{array}{c}\text { Entapa 1 } \\
\text { Sensibilización/ } \\
\text { exploridad }\end{array}$} & $\begin{array}{c}\text { Etapa 2 } \\
\text { adopción/aplicación } \\
\text { rápida }\end{array}$ & $\begin{array}{r}\text { Etapa 3 } \\
\text { experiencia en } \\
\text { la aplicación/ } \\
\text { crecimiento }\end{array}$ \\
\hline UAO & & & & & & \\
\hline UAB & & & & & & \\
\hline UB & & & & & & \\
\hline UdG & & & & & & \\
\hline UdL & & & & & & \\
\hline UVic-UCC & & & & & & \\
\hline UIC & & & $\mathrm{NC}^{*}$ & & & \\
\hline UOC & & & $\mathrm{NC}^{*}$ & & & \\
\hline UPC & & & & & & \\
\hline UPF & & & & & & \\
\hline URL & & & & & \\
\hline URV & & & & & & \\
\hline
\end{tabular}

${ }^{*} \mathrm{NC}=$ No contestada

Fuente: Elaboración propia según los resultados.

En este estado de desarrollo, 5 universidades se autodefinen en etapas iniciales exploratorias o de escaso desarrollo de la SP (etapas 1 o 1-2), mientras que otras $5 \mathrm{se}$ 
declaran en fases más maduras y consolidadas de implantación de la SP (etapas 2 a 3) y 2 no contestan, por considerar que no pertenecen a ninguna etapa al no tener implantado ni previsto ningún grado SP. La opinión general es que no existe una reclamación de más grados en modalidad SP, excepto en 2 universidades privadas y una pública.

Las infraestructuras tecnológicas, que deben dar apoyo a la docencia SP, se declaran en general suficientes, aunque las universidades grandes (UB, UAB) y la URL reconocen que existen limitaciones y la capacidad de servidores y anchos de banda son valorados de manera muy heterogénea, probablemente por el desarrollo todavía incipiente (número total de alumnos que cursan en SP) de la demanda.

La evaluación de los grados SP tiene lugar normalmente dentro de los programas generales preceptivos de verificación, seguimiento, acreditación y certificación de la AQU. En algunos casos son objeto de evaluación más detallada, aunque poco formalizada.

No suele requerirse un perfil específico al profesorado que imparte SP y, en general, las universidades ofrecen a los profesores un apoyo genérico de índole tecnológica y pedagógica. El reconocimiento de la tarea docente del profesorado de SP es, en la mayoría de las universidades, idéntico al de la docencia en modalidad presencial; aun admitiendo en la mayoría de universidades una carga superior, tan sólo tres de ellas reconocen explícitamente una dedicación superior (30-50\%). En el capítulo de incentivos, mayoritariamente no se declara ningún estímulo en los diversos ítems de la entrevista. De las 12 universidades consultadas, en tres casos se ofrecen becarios de apoyo; en dos universidades una reducción de la carga docente; en una universidad una compensación económica para la producción de materiales docentes y, finalmente en otra universidad el reconocimiento de méritos suplementarios en convocatorias de innovación docente.

\section{Causas que explican la situación actual}

Los resultados del segundo bloque de respuestas (ítems 1-15) se muestran en la Figura 2 y representan el análisis de los responsables académicos sobre qué factores influyen en la escasa implantación de la SP. La UOC no respondió al tratarse de una universidad no presencial. 
Figura 2. Box-Plot con los resultados de las respuestas a las preguntas 1-15 del cuestionario a vicerrectores. $\mathrm{X}=$ media; caja $=\mathrm{DS}$; límites de la barra: intervalo de respuestas

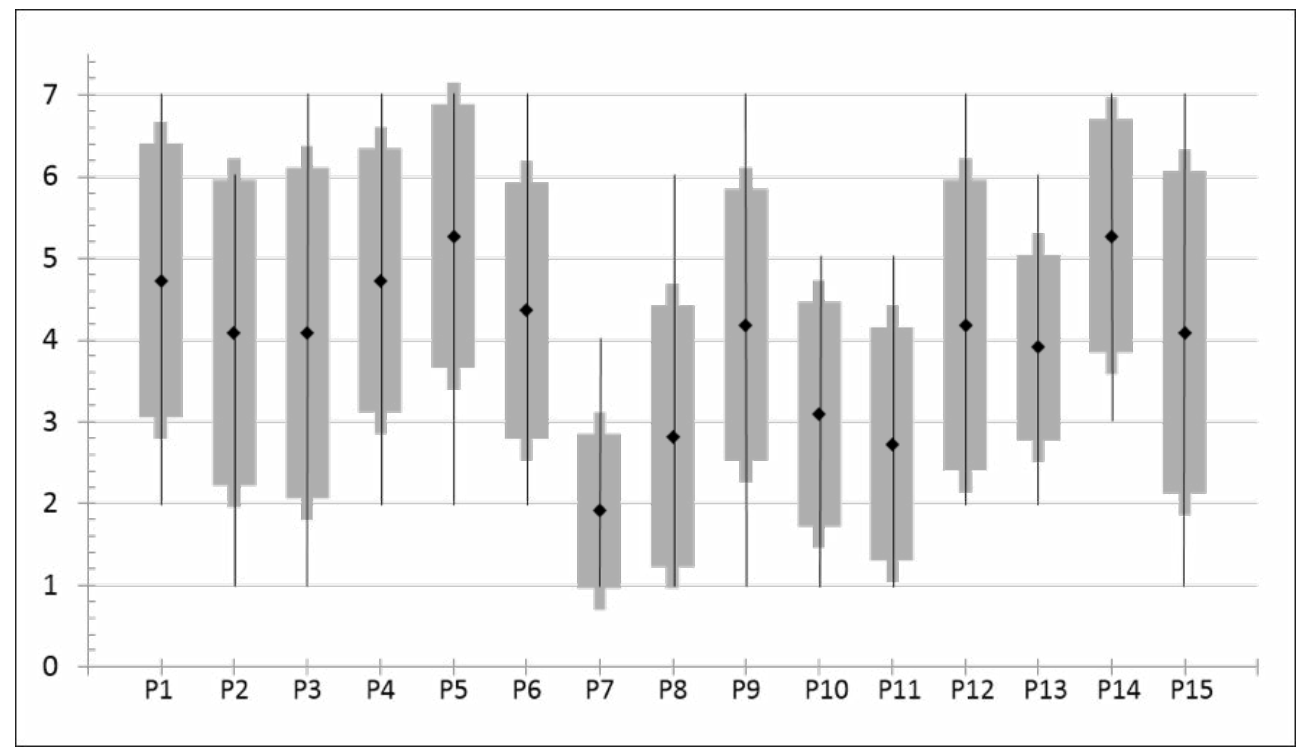

P1 = Normativa de la AQU muy restrictiva y poco flexible; P2 = Directrices de la institución para promover el AC poco definidas; $\mathrm{P}_{3}$ = Escasa demanda desde el punto de vista social o de los estudiantes; $\mathrm{P} 4$ = Poca tradición de cooperación interuniversitaria para realizar Grados AC compartidos; $\mathrm{P}_{5}=$ Falta de recursos económicos suficientes para implementar el AC correctamente; P6 = Inseguridad del profesorado ante algunas políticas suprauniversitarias (por ejemplo: derechos de propiedad intelectual, Ley de protección de datos, copyright, etc.); $\mathrm{P}_{7}=$ Espacios virtuales disponibles en la institución lentos, cerrados o poco adecuados al AC; P8 = Espacios físicos para desarrollar nuevas actividades relacionadas con el AC poco adecuados o insuficientes; $\mathrm{P} 9$ = Reconocimiento y carga docente igual para todas las modalidades de enseñanza; P10 = Disponibilidad de soporte técnico limitada y no específica; P11 = Disponibilidad de apoyo pedagógico limitada y no específica; P12 = Formación del profesorado inexistente o no específica; $\mathrm{P} 13$ = Becarios de apoyo para el primer año de la implementación del AC inexistentes o insuficientes; P14 = Poco estímulos a todos los niveles para el profesorado para impartir AC; P15 = Alumnado poco o nada predispuesto a esta modalidad en los primeros cursos universitarios.

Fuente: Elaboración propia a partir de los resultados.

Cabe destacar la gran dispersión de respuestas, indicativa de la atribución y valoración de causas muy distintas como determinantes de la situación actual y en función de la tipología de universidad, estudios ofrecidos, intentos anteriores, potencialidad del profesorado, necesidades objetivas, planificación estratégica, etc. La mayor coincidencia ( 1 o 2 discrepancias) se da en los ítems 5 (insuficientes 
recursos económicos para implantar la SP, valorada como relevante, entre 4-7); 7 (limitaciones en la capacidad de los espacios virtuales para desarrollar la SP, valorada como poco influyente, 1-2); 9 (reconocimiento insuficiente de la carga docente suplementaria que representa la SP, considerada como moderadamente influyente, 4-5) y 14 (estímulos insuficientes al profesorado para impartir en modo SP, bastante influyente, 4-6). Un segundo bloque de temas con un menor grado de coincidencia (3 discrepancias) señalaría todavía como causas relevantes las correspondientes a los ítems 4 (poca explotación de la creación de grados SP compartidos; bastante o muy de acuerdo, 4-7) y 6 (inseguridad del profesorado frente a aspectos legales y de contexto del trabajo en internet; bastante o muy de acuerdo, 4-7).

Los matices cualitativos manifestados por los vicerrectores en las entrevistas perfilaron percepciones realmente distintas de las posibles limitaciones en cada universidad, sin patrones de agregación por tipología de universidad, tipo de grados propuestos, implantación territorial ni de ninguna otra ensayada, por lo que se asume que la opinión de cada universidad responde realmente a su propia experiencia y, en todo caso, la dispersión de resultados debe relacionarse también con el escaso número de casos analizados, al haberse desplegado en muchos casos un número muy bajo o nulo de grados SP.

\section{Perspectivas futuras del aprendizaje combinado}

En último lugar, se preguntó a todos los vicerrectores por su opinión hacia donde avanza, o debería progresar, el AC en un horizonte cercano de los próximos 3 años. Todos los vicerrectores, sin excepción, manifiestan su apuesta para maximizar las posibilidades de sus respectivos Campus Virtuales y fomentar la combinación de metodologías activas en el aula haciendo uso del abanico de posibilidades asociadas al uso intensivo de las tecnologías.

En este contexto, algunos vicerrectores, que coinciden con las universidades más pequeñas y las privadas, indican que este es el camino hacia modalidades híbridas, más allá del cómputo de horas presenciales de los estudiantes en el aula. La UPF indica que la modalidad predominante en su formación es, justamente, este AC atendiendo al uso intensivo de la tecnología en la mayor parte de sus asignaturas.

Hay coincidencia total en la importancia de la interacción intensiva profesorestudiante de las universidades presenciales en alumnos jóvenes que provienen del bachillerato. Es por ello que no se cree necesario ni conveniente reducir el número de horas presenciales, a menos que se den circunstancias especiales que así lo aconsejen (tasas altas de repetidores, dobles titulaciones, etc.). Mención aparte merecen algunas nuevas titulaciones muy profesionalizantes que, al dirigirse a un sector de estudiantes mayor, a menudo dentro de mercado de trabajo, tengan difícil su asistencia diaria. Así, algunas universidades (UdG, UdL, URL, UdV) apuntan que están preparando a corto plazo propuestas de cambio de modalidad (de presencial a 
semipresencial reglada) de grados ya existentes o, de otros nuevos que necesitan aún la aprobación de la AQU.

Existe más disparidad de opiniones en cuanto a la necesidad de orientar a los docentes una reducción de clases expositivas, para promover más actividades dentro y fuera del aula que pudieran conducir progresivamente a un aprendizaje más" híbrido". Los argumentos son variados, pero destacan como dificultades para esta disminución: 1) la tipología y singularidad de cada disciplina, 2) la edad de los docentes (muy elevada en las universidades más grandes) y 3) el contexto de crisis general dentro de la universidad (más carga docente por falta de reposición del profesorado jubilado). Las universidades privadas y públicas más jóvenes (UPF, URV) afirman tener un mayor control y seguimiento sobre las acciones de sus docentes y las actividades que estos realizan dentro y fuera del aula.

En todos los casos, se admite que, de manera voluntaria, ya se están realizando "mezclas" de diferentes modalidades de aprendizaje en muchos grados pero, en pocos casos, se reconoce que se promuevan iniciativas con una clara orientación hacia la modalidad semipresencial acreditada. Se apuesta más por los cursos masivos MOOCs (aunque admiten su poca relación con las modalidades híbridas) que con elementos de Flipped Classroom (aula invertida), si bien a menudo se constatan iniciativas de este último tipo como formas de aprendizaje más activas que superan la clase tradicional.

Por último, se preguntó sobre el uso de los datos generados por los recursos de los Campus Virtuales propios y su potencial utilización en la mejora del aprendizaje (Learning Anaylitics). Excepto la universidad virtual UOC, en el resto de casos no constaba el uso de datos extraídos de las herramientas propias de la universidad, con la excepción de las encuestas de satisfacción que, en mayor o menor grado, todas las universidades promueven y cuya utilidad remarcaba especialmente la UB. Aunque, en general, los responsables académicos conocen el potencial futuro del Learning Analytics, Big Data y/o Adaptative Learning para la mejora de los aprendizajes ligados a modalidades híbridas; todos coinciden en que el uso de estos datos no va a poder ser explotado de manera extensiva, atendiendo a las normativas de protección de los datos de los estudiantes.

\section{DISCUSIÓN Y CONCLUSIONES}

\section{Implantación y desarrollo de la semipresencialidad reglada}

Para obtener un marco global de análisis, se consultaron los últimos informes HORIZON de 2014 a 2017 de Educación Superior, los Innovating Pedagogy Report de la Open University, así como documentos de trabajo de las jornadas Future of Blended Learning organizadas por la LERU (League of European Research Universities). Los datos aportados sobre implantación de grados en modalidad semipresencial reglada (SP) demuestran que esta no es significativa actualmente 
(2016-2017) en las universidades estudiadas, aún a pesar del crecimiento pronosticado hace cinco años (Simon et al., 2013). Su limitada extensión (3,7\% del total de grados impartidos) define un ámbito de aplicación muy restringido y especializado: algunas universidades, pequeñas, privadas, que disponen así de una solución estratégica para la gestión de su demanda formativa, junto a otros pocos casos muy concretos que permiten dar respuesta a problemáticas específicas, en general de corto alcance temporal, fundamentalmente aplicada al ámbito de las ciencias sociales y, en menor grado, a las ciencias de la salud. En cambio, la modalidad semipresencial tiene un mayor desarrollo en másteres para el cual se revela más adaptado, y para las mismas ramas de conocimiento.

Las universidades grandes, públicas, con más del $80 \%$ de la oferta de grados, se definen (y se reivindican) como presenciales. Ahora bien, reconocen la conveniencia (que desarrollan en la práctica diaria) de orientar una parte del aprendizaje hacia estrategias de todo tipo (basadas a menudo, pero no exclusivamente, en el uso de tecnologías) fuera del contexto de las sesiones magistrales (out classroom, pero ni tan siquiera out campus!), es decir, en una reducción parcial de la presencialidad clásica, en la línea del AC que, en algún momento, se ha denominado también de semipresencialidad, introduciendo cierta confusión conceptual o terminológica puesto que nada tiene que ver con la semipresencialidad reglada.

Las causas de su escasa implantación -a pesar del potencial que permitía su cobertura legal- pueden deducirse de varias respuestas de los encuestados, pero son muy significativos los resultados a los ítems 1-15 representados en la Figura 1: la gran dispersión de las respuestas indicaría que ninguno de los factores sugeridos en la encuesta (derivados de los identificados por Porter et al., 2016) es determinante de manera clara en la no-implantación de la SP. Según nuestra opinión, debemos atribuirla a una causa estratégica previa (o mejor, a su ausencia), es decir: no ha dejado de desplegarse la SP por el fracaso de uno u otro determinante, sino por la ausencia de decisión a su favor, implícitamente defendiendo el carácter presencial mayoritario. También apunta en la misma dirección que las universidades que han considerado la SP como opción estratégica, al menos en parte de su oferta (principalmente pequeñas y privadas) han conseguido duplicar su oferta en 5 años, siguen considerándola válida y satisfactoria y se autodefinen en etapas consolidadas de su implantación (etapas 2-3, $f f$. Tabla 5). No se debe interpretar, por tanto, como un fracaso, sino como una opción estratégica de uso restringido, hacia una tipología muy concreta de enseñanzas.

\section{Desarrollo del aprendizaje combinado (Blended Learning)}

Se constata el problema de conceptualización del Blended Learning, como lo demuestra la consideración que, en muchos de los grados formalmente presenciales, ya se está incluyendo parcialmente este aprendizaje, aunque sea de forma poco explícita. En todas las universidades se asegura que, en un futuro, este modelo 
estará básicamente circunscrito a los másteres por una cuestión meramente de compatibilización y flexibilización horaria.

Se han identificado unos patrones comunes que permiten definir las tipologías (figura 3) para interpretar la evolución del AC de manera explícita. La mitad de los participantes $(50 \%)$ dio una definición básica genérica de «mezcla de enseñanza presencial con fuerte soporte TIC», un tercio (33,3\%) dio una visión normativizada de actividades en línea con alguna sesión presencial. Sólo unos pocos $(16,6 \%)$ proporcionaron tipos de definiciones más calificadas «el aprendizaje combinado es una combinación explícita de metodologías mediadas por las TIC», y un caso $(8,3 \%)$ el entrevistado no se pronunció atendiendo a que el término blended (o cualquiera de sus acepciones) se considera conceptualmente inadecuado. En general, los porcentajes no se pueden correlacionar con la tipología de las universidades.

Figura 3. Esquema de tipologías del Blended Learning en el panorama universitario catalán

\section{ENFOQUE}

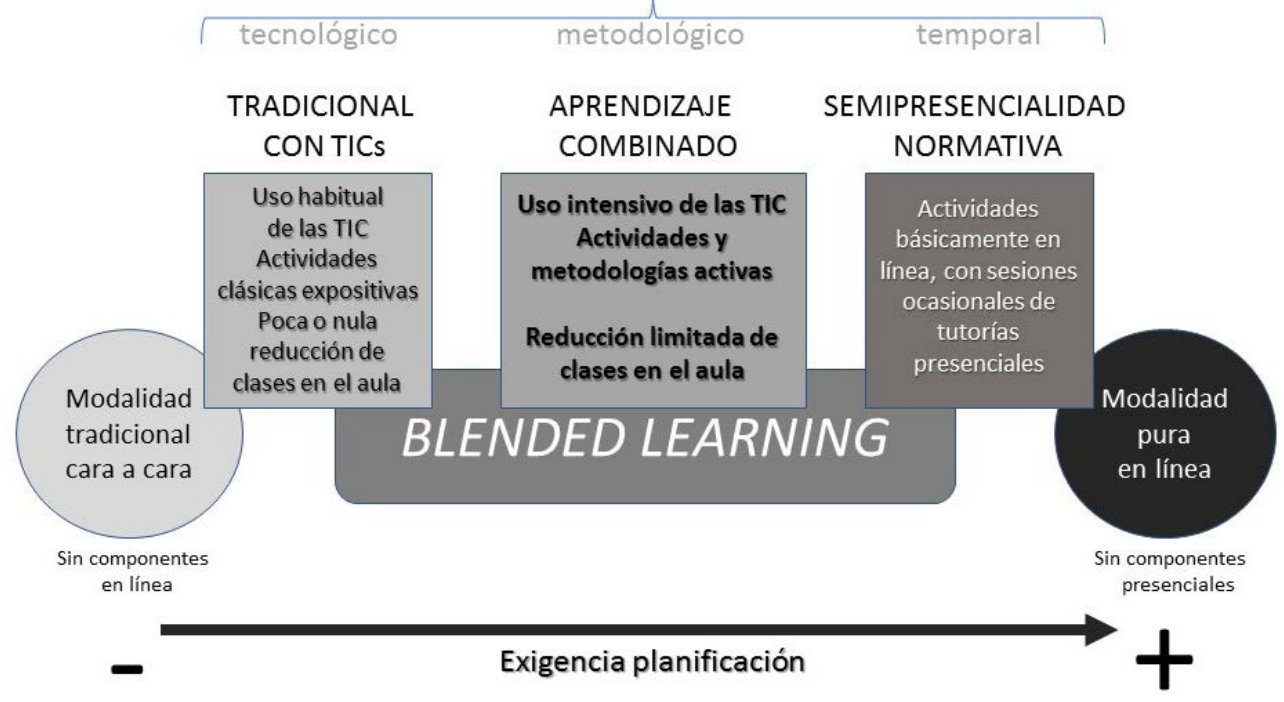

Fuente: Elaboración propia a partir de los resultados.

En todas las universidades hay coincidencia en que el aprendizaje combinado puede ser útil, conceptualizándolo de forma generalista y contraponiéndolo tanto al e-learning como a la semipresencialidad normativizada por la AQU. Aproximadamente un tercio de los casos opinan que el aprendizaje combinado sensu lato tiene un gran potencial para mejorar el aprendizaje a nivel de grado. En este sentido, se hace hincapié que el profesorado se esfuerza por mejorar las experiencias de aprendizaje y el éxito académico. 
El acceso a materiales y tecnología de aprendizaje no es un problema para los estudiantes de las universidades catalanas, atendiendo a que todas ellas disponen de campus virtuales plenamente funcionales e infrautilizados desde el punto de vista metodológico. Se deduce de las respuestas, de la existencia de un problema en la formación técnico-pedagógica de buena parte del profesorado, si bien todas las universidades dedican personal y medios a su formación.

No se puso de relieve como factor limitante para la implementación del AC el aumento de la carga docente y la posible limitación en habilidades tecnológicas y metodológicas limitadas en profesorado senior. Sí se consideró importante el reconocimiento ecuánime del esfuerzo de los equipos docentes, así como la formación en habilidades pedagógicas y tecnológicas. Estos dos aspectos coinciden con la revisión del Grupo de Oxford (2013) y con los resultados de Simon et al. (2016). Sorprendentemente, los entrevistados no consideraron crítico el papel del estudiante, si bien Kenney y Newcombe (2010) identificaron como un claro desafío en el despliegue del AC, la participación de los estudiantes, su familiaridad con la tecnología y la autodisciplina frente al estudio.

\section{Algunas sugerencias finales}

A modo de conclusión, aportamos unas ideas-clave o propuestas de reflexión para la implementación exitosa del aprendizaje combinado:

1. Llevar a cabo un análisis de las necesidades reales en relación con la visión y misión de cada universidad y los mecanismos de apoyo necesarios antes de diseñar cursos en esta modalidad.

2. Seleccionar cuidadosamente el modelo de aprendizaje combinado que sea más adecuado para la institución y con reconocimiento previo de las limitaciones de la misma.

3. Proporcionar formación continua al personal docente -incluyendo personal administrativo y de apoyo- para conducir el programa y mejorar continuamente la efectividad del aprendizaje.

4. Crear un sistema efectivo de soporte técnico para profesorado y alumnado, también para hacer frente a los posibles fallos tecnológicos.

5. Alentar a los profesores a trabajar en colaboración entre sí mediante la creación de equipos docentes para compartir ideas y/o mejores prácticas.

6. Reconocer, de forma adecuada y suficiente, la carga docente en el aprendizaje combinado. 


\section{Agradecimientos}

Nuestra gratitud a los vicerrectores y vicerrectoras de las doce universidades que han participado en el estudio, por la atención, buena disposición y gran cordialidad en las entrevistas personales.

Al Instituto de Ciencias de la Educación (ICE) de la UB por la financiación del proyecto REDICE16-1580, y al Jefe de su Sección de Investigación, Dr. Joan Mateo, por el asesoramiento en la reorientación del proyecto.

Al Dr. Antonio R. Bartolomé, del departamento de Didáctica y Organización Educativa de la UB, por habernos invitado y animado en participar en este número monográfico de RIED.

A la Sra. Núria Comet Señal, Coordinadora de Proyectos y Responsable de Calidad Interna de la AQU, por facilitarnos el informe ROC.

Al Sr. Francisco Pérez del Instituto de Formación Continuada IL3 de la UB por su colaboración en el proyecto.

Finalmente, al Vicerrectorado de Política Docente de la UB y de manera destacada a su vicerrectora Dra. Amelia Díaz, por su ayuda y apoyo incondicional durante el estudio.

\section{NOTAS}

1. Relación de vicerrectores/as entrevistados en enlace externo

2. Modelo de entrevista disponible en enlace externo

3. Detalles agregados de grados por universidades y modalidad disponibles en enlace externo

4. Detalles agregados de másteres por universidades y modalidad disponibles en enlace externo

\section{REFERENCIAS BIBLIOGRÁFICAS}

Abrahams, D.A.(2010). Technology adoption in higher education: A framework for identifying and prioritizing issues and barriers to adoption of instructional technology.JournalofApplied Researchin Higher Education 2.2, 34-49. doi: http:/ dx.doi.org/10.1108/17581184201000012

Adams Becker, S., Cummins, M., Davis, A., Freeman, A., Hall Giesinger, C., y Ananthanarayanan, V. (2017). NMC Horizon Report: 2017 Higher Education Edition. Austin, TX: The New Media Consortium.
Alebaikan, R., y Troudi, S. (2010). Blended learning in Saudi universities: challenges and perspectives. ALT-J Research in Learning Technology, 18(1). 49-59. doi: http://dx.doi. org/10.1080/09687761003657614

AQU Catalunya. (2016). Guia per $a$ l'acreditació de les Titulacions Universitàries Oficials de Grau i Màster. Barcelona: Agència per a la Qualitat del Sistema Universitari de Catalunva. Recuperado de http://www.aqu.cat/doc/ doc 22567776 1.pdf 
Bartolomé, A. (2004). Blended Learning. Conceptos básicos. Píxel-Bit. Revista de Medios y Educación, 23, 7-20.

Benítez, R., y Aguilar, S. (2010). Situación del B-learning en las diferentes universidades públicas andaluzas. Edutec-e, Revista Electrónica de Tecnoloqía Educativa. Recuperado de http://www.edutec.es/ revista/index.php/edutec-e/article/ download/441/176

Brooks, L. (2008). An analysis of factors that affect faculty attitudes toward a blended learning environment. Ph.D dissertation, Faculty of the College of Education, TUI University, California.

Dziuban, C. D. (2015). Big data in online and blended learning research. Conducting Research in Online and Blended Learning Environments: New Pedagogical Frontiers, 143.

Eddy, L. J., Nor-Aziah, A., y Jasmine, J. (2014). Blended Learning: Examining Concepts and Practices. Blended \& Flipped Learning: Case Studies in Malaysian HEIs. Bangi: Pusat Pengajaran \& Teknologi Pembelajaran, Universiti Kebangsaan Malaysia.

Entwistle, N., y Ramsden, P. (2015). Understanding Student Learning (Routledge Revivals). Oxon: Routledge.

Expósito, E., yTurull, M.(2014).Experiencias de docencia semipresencial en el título de Derecho. Barcelona: Edicions Universitat Barcelona.

Future of Blended learning (2015). LERU e-learning Working Group - Utrech. Recuperado de http://www.leru.org/ index.php/public/home/

Garrison, D. R., y Kanuka, H. (2004). Blended learning: Uncovering its transformative potential in higher education. The Internet and Higher Education, 7(2), 95-105.

Garrison, D. R., y Vaughan, N. D. (2007). Blended learning in higher education: Framework, principles, and guidelines. San Francisco, CA: Jossey-Bass.
Gomez, J., e Igado, M. (2008). Blended learning: The key to success in a training company. International Journal of Instructional Technology and Distance Learning, 5(8). Recuperado de http:/ www.itdl.org/Journal/Aug 08/article04. htm

Graham, C. R., Allen, S., y Ure, D. (2005). Benefits and challenges of blended learning environments. En M. KhosrowPour (Ed.), Encyclopedia of Information Science and Technology, (253-259). Hershey, PA: Idea Group Inc.

Graham, C. R. (2006). Blended learning systems: Definition, current trends, and future directions. En C. J. Bonk y C. R. Graham (Eds.), Handbook of blended learning: Global perspectives, local designs (3-21). San Francisco, CA: Pfeiffer Publishing.

Graham, C. R., Woodfield, W., y Harrison, J. B. (2012). A framework for institutional adoption and implementation of blended learning in higher education. The Internet and Higher Education, 18, 4-14.

Graham, C. R. (2013). Emerging practice and research in blended learning. En M.G. Moore (Ed.), Handbook of distance education ( $3^{\mathrm{a}}$ ed.) (333-350). New York, NY: Routledge.

Halverson, L. R., Graham, C. R., Spring, K. J., Drysdale, J. S., y Henrie, C. R. (2014). A thematic analysis of the most highly cited scholarship in the first decade of blended learning research. The Internet and Higher Education, 2O, 20-34.

Horizon (2017). NMC Informe Horizon Edición de Educación Superior. Austin, Texas: The New Media Consortium. Recuperado de http://www.nmc.org nmc-horizon

Humbert, M. (2007). Adoption of blended learning by faculty: an exploratory analysis. En M. K. McCuddy (Ed.), The challenges of educating people to lead in a challenging world (423-436). Dordrecht: Springer. 
Innovating Pedagogy (2017). Institute of Educational Technology. Open University Recuperado de http://www.open.ac.uk/ blogs/innovating

Kenney, J., y Newcombe, E. (2010). Adopting a blended learning approach: Challenges encountered and lessons learned in an action research study. Journal of Asynchronous Learning Networks, 15(1), 45-57.

Kim, K. J., Bonk, C. J., y Oh, E. (2008). The present and future state of blended learning in workplace learning setting in the United States. Performance Inprovement, 47(8), 5-16. doi: http:// dx.doi.org/10.1002/pfi.20018

Korr, J., Derwin, E. B., Greene, K., y Sokoloff, W. (2012). Transitioning an AdultServing University to a Blended Learning Model. The Journal of Continuing Higher Education, 60, 2-11. doi: http://dx.doi.or g/10.1080/07377363.2012.649123

Moskal, P., Dziuban, C., y Hartman, J. (2013). Blended learning: A dangerous idea? The Internet and Higher Education, $18,15-23$.

Norberg, A., Dziuban, C., y Moskal, P. D. (2011). A time-based blended learning model. On the Horizon, 19(3), 207-216.

Oh, E., y Park, S. (2009). How are universities involved in blended instruction? Educational Technology \& Society, 12(3), 327-342.

Osguthorpe, R. T., y Graham, C. R. (2003). Blended learning environments: Definitions and directions. Quarterly Review of Distance Education, 4(3), 227234.

Owston, R., York, D., y Murtha, S. (2013). Student perceptions and achievement in a university blended learning strategic initiative. The Internet and Higher Education, 18, 38-46. doi: http://dx.doi. org/10.1016/j.iheduc.2012.12.003

Porter, W. W., Graham, C. R., Spring, K. A., y Welch, K. R. (2014). Blended learning in higher education: Institutional adoption and implementation. Computers \& Education, 75, 185-195.

Porter, W. W., y Graham, C. R. (2015). Institutional drivers and barriers to faculty adoption of blended learning in higher education. British Journal of Educational Technologu. doi: http:// dx.doi.org/10.1111/bjet.12269

Porter, W. W., Graham, C. R., Bodily, R., y Sandberg, D. (2016). A qualitative analysis of institutional drivers and barriers to blended learning adoption in higher education. Internet and Hiqher Education, 28(1), 17-27. doi: http:/ dx.doi.org/10.1016/j.iheduc.2015.08.003

ROC (2016). Registre Oficial de Centres $i$ Estudis Universitaris a Catalunya. Secretaria d'universitat i Recerca. Generalitat de Catalunya.

Ross, B., y Gage, K. (2006). Global perspectives on blended learning: Insight from WebCT and our customers in higher education. En C. J. Bonk y C. R. Graham (Eds.), Handbook of blended learning: Global perspectives, local designs (155168). San Francisco, CA: Pfeiffer.

Simon, J., Benedí, C., y Blanché, C. (2013). La semipresencialidad como respuesta a los nuevos retos de la universidad: la visión de las universidades de la Xarxa Vives. Barcelona: Octaedro.

Simon, J., Benedí, C., Blanché, C., y Bosch, M. (2016). La semipresencialidad en Educación Superior: casos de estudio en los grados de la universidad de Barcelona. Edutec. Revista Electrónica de Tecnología Educativa, 58: 15-33.

Spring, K. J., Graham, C. R., y Hadlock, C. A. (2016). The current landscape of international blended learning. International Journal of Technology Enhanced Learning, 8(1), 84-102.

The Oxford Group. (2013). Blended Learning-Correct Use, Challenges and Best Practices, Report 2013. Kineo.

Waha, B., y Davis, K. (2014). University students' perspective on blended learning. 
Journal of Higher Education Policy and Management, 36(2), 172-182.

Yamagata-Lynch, L. C. (2014). Blending online asynchronous and synchronous learning. The International Review of Research in Open and Distributed Learning, 15(2), 189-212. doi: http:// dx.doi.org/10.19173/irrodl.v15i2.1778.

\section{PERFIL ACADÉMICO Y PROFESIONAL DE LOS AUTORES}

Joan Simon Pallisé. Profesor titular de la Facultad de Farmacia de la Universidad de Barcelona, con Máster Internacional en e-Learning (Especialidad en Dirección), Máster oficial en Educación y TIC y el Postgrado: Redes sociales e Intercambio del conocimiento. Coordinador del Grupo de Innovación Docente GIBAF de la UB, ha obtenido dos distinciones Jaume Vicens Vives a la calidad docente universitaria. Sus líneas de investigación se centran en el Aprendizaje servicio, docencia semipresencial, entornos personales de aprendizaje y evaluación continuada.

E-mail: joansimon@ub.edu

Carles Benedí González. Profesor titular de la Facultad de Farmacia de la Universidad de Barcelona. Miembro del Grupo de Innovación Docente GIBAF de la UB, ha obtenido una distinción Jaume Vicens Vives a la calidad docente universitaria. Sus líneas de investigación se centran en el Aprendizaje servicio, docencia semipresencial, entornos personales de aprendizaje y evaluación continuada.

E-mail: cbenedi@ub.edu

Cèsar Blanché Vergès. Profesor catedrático de la Facultad de Farmacia de la Universidad de Barcelona, miembro del Grupo de Innovación Docente GIBAF de la UB ha obtenido una distinción Jaume Vicens Vives a la calidad docente universitaria. Sus líneas de investigación se centran en el Aprendizaje servicio, docencia semipresencial, entornos personales de aprendizaje y evaluación continuada.

E-mail: cesarblanche@ub.edu

Maria Bosch Daniel. Profesora titular de la Facultad de Farmacia de la Universidad de Barcelona, miembro del Grupo de Innovación Docente GIBAF de la UB, ha obtenido una distinción Jaume Vicens Vives a la calidad docente universitaria. Sus líneas de investigación se centran en el Aprendizaje servicio, docencia semipresencial, entornos personales de aprendizaje y evaluación continuada. E-mail: mariabosch@ub.edu 


\section{DIRECCIÓN DE LOS AUTORES}

Departamento de Biología, Sanidad y Medio Ambiente

Facultad de Farmacia

Av. Joan XXIII, 27-32

o8028 Barcelona

España

Mercedes Torrado Fonseca. Profesora titular de la Facultad de Educación. Pertenece al equipo de investigación TRALS (Transiciones Académicas y Laborales) del departamento de Metodología de Investigación, y mimbro de la Asociación Interuniversitaria de Investigación Pedagógica. Sus ámbitos de especialización están relacionados con la investigación educativa, en especial con el análisis de datos, la aplicación de técnicas de estadística multivariante con el programa SPSS, y la elaboración de instrumentos de evaluación. Las líneas de investigación son: transiciones académicas, inserción laboral, tutoría universitaria y rendimiento académico.

E-mail: mercedestorrado@ub.edu

\section{DIRECCIÓN DE LA AUTORA}

Departamento de Métodos de Investigación y Diagnóstico en Educación

Facultad de Educación

Paseo de Vall d'Hebron, 171

o8035 Barcelona - España

Fecha de recepción del artículo: 07/04/2017

Fecha de aceptación del artículo: 31/05/2017

\section{Como citar este artículo:}

Pallisé, J. S., Benedí González, C., Blanché Verges, C., Bosch Daniel, M., y Torrado Fonseca, M. (2018). Análisis cuantitativo y cualitativo de la semipresencialidad del sistema universitario de Cataluña. RIED. Revista Iberoamericana de Educación a Distancia, 21(1), pp. 113-133. doi: http://dx.doi.org/10.5944/ried.21.1.18773 